\title{
ÍNDICE
}

Págs.

INTRODUCCIÓN

7

G. Montero González, F. Bravo Oviedo y F.J. Silvia-Pando: NOTAS SOBRE

INVESTIGACION FORESTAL: UNA PERSPECTIVA SELVÍCOLA Y DE GESTIÓN

9

\section{CONFERENCIAS Y PONENCIAS}

E. ROJAS BRIALES: LOS RETOS DE LA HUMANIDAD ANTE LA PERSPECTIVA DE 2050 Y LOS BOSQUES

Ma GAFO GÓMEZ-ZAMALlOA: POLÍTICA FORESTAL Y DESARROLLO RURAL:

VISIÓN DESDE LA UE

P. CAMPos Palacín: RENTA AMBIENTAL DEL MONTE 35

J.A. VEGA HidALGO: NUEVOS RETOS Y OPORTUNIDADES DE MEJORA EN

LA LUCHA CONTRA INCENDIOS FORESTALES Y SUS CONSECUENCIAS 73

M.Ma ${ }^{\mathrm{a}}$ RUIZ URRESTARAZU: NOMBRES Y PAISAJE

F. SEVILLA MARTíneZ: LA PREDICCIÓN EN SISTEMAS COMPLEJOS: CIENCIA Y APLICACIÓN PRÁCTICA

F. BRAVO OVIEDO: ¿SE PUEDE GENERAR CONOCIMIENTO MEDIANTE LA GESTIÓN ADAPTATIVA PARA FUNDAMENTAR UN CAMBIO DE PARADIGMA DE LA SELVICULTURA?

P. Fernández Rebollo, Ma .D. CARbonero MuÑoz y A. García Moreno: CONTRIBUCiÓN DE LA GANADERÍA EXTENSIVA AL MANTENIMIENTO DE LAS FUNCIONES DE LOS ECOSISTEMAS FORESTALES

R. SIERRA DE GRADO: MEJORA GENÉTICA: LO QUE EL VIENTO NOS TRAERÁ

J. PemÁn García: LECCIONES APRENDIDAS Y TAREAS PENDIENTES EN LA ACTIVIDAD REPOBLADORA Y VIVERÍSTICA 
F. TORRENT BRAVO: REFLEXIONES SOBRE LA GESTIÓN DE PESCA DEPORTIVA ACTUAL EN ESPAÑA

C. Martínez Santa-María: EL AGUA EN EL AULA: ¿CÓMO ENSEÑAR SU NUEVA DIMENSIÓN?

J. DE LAS HERAS IBÁÑEZ: ¿QUÉ HACER DESPUÉS DEL FUEGO? PRINCIPALES RETOS

Y ALGUNAS RESPUESTAS

C. HERNANDO LARA: PREVENCIÓN DE INCENDIOS FORESTALES:

¿QUÉ ESTUDIAMOS? ¿CÓMO TRANSFERIMOS EL CONOCIMIENTO?

J.J. DieZ CASERO: PATOLOGÍAS FORESTALES Y CAMBIO GLOBAL:

GLOBALIZACIÓN, CAMBIO CLIMÁTICO Y CUESTIONES LEGALES

R. Zas ArRegui y L. SAMPEdRo PÉREZ: RESISTENCIA DE LOS PINOS A PLAGAS Y ENFERMEDADES: NUEVAS OPORTUNIDADES DE CONTROL FITOSANITARIO

A. Rojo Alboreca: EL RETO DE LA ORDENACIÓN DE LOS MONTES PRIVADOS EN ESPAÑA

M. PiQué NicOlaU: PLANIFICACIÓN FORESTAL EN ESPACIOS NATURALES

PROTEGIDOS: HERRAMIENTAS INTEGRADORAS EN UN CONTEXTO DE CAMBIO . 299

F. DE JUANA ARANZANA: GESTIÓN DE ZONAS VERDES URBANAS Y PERIURBANAS

PARA LA CONSERVACIÓN DE LA BIODIVERSIDAD: EL CASO DE VITORIA-GASTEIZ.. 313

A. Vega Cueto, E. Canga Libano, S. Sánchez García, A. Hevía Cabal, I. Feito Díaz, Ma Menéndez-Miguélez, M. González-García, C. Martínez Alonso y J.P. Majada GuiJo: INNOVACIÓN DE PROCESOS Y PRODUCTOS EN EL SECTOR DE LA MADERA

J.I. Fernández-Golfín Seco y E. Hermoso Prieto: LAS NECESIDADES DE I+D+i Y DE FORMACIÓN DE LA INDUSTRIA PRIMARIA DE LA MADERA EN ESPAÑA

L. Díaz BALTEIRO: ASPECTOS ECONÓMICOS DEL RECURSO MICOLÓGICO.

IMPLICACIONES PARA LA GESTIÓN FORESTAL

J. PICOS MARTÍN: INDUSTRIA FORESTAL Y MERCADO DE LA MADERA: NAVEGANDO

EN LA TORMENTA 367

CONCLUSIONES DEL $6^{\circ}$ CONGRESO FORESTAL ESPAÑOL 381 